Sir,

\section{Choroidal effusion after YAG laser capsulotomy}

Choroidal effusion can occur within 1 to 3 days following various ocular and intraocular surgical procedures such as cataract and retinal detachment surgery, ${ }^{1}$ even in the absence of a wound leak, as well as after retinal laser and cryocoagulation. ${ }^{2,3}$ It is assumed that in most cases choroidal effusion is initiated by post-operative intraocular inflammation. ${ }^{1}$

We present an unusual case of choroidal effusion after capsulotomy performed with a neodymium-YAG laser.

\section{Case report}

In 1995 uneventful phacoemulsification with implantation of a Morcher Type 66 lens (+16 D) into the posterior chamber was performed in the left eye of a 79-year-old patient. Pre-operatively a moderate myopia was noticed in both eyes, $-3.5 /-1.5 / 90^{\circ}$ OD and -4.5 DS (spherical dioptres) OS. The visual acuity improved from 0.3 to $\left(-2.0 /-1.0 / 0^{\circ}\right) 0.5$ post-operatively, but

deteriorated to 0.2 due to regenerative secondary cataract in October 1997. After an uncomplicated surgical polishing of the posterior capsule a recurrence of the secondary cataract was seen in February 1999, which led to a decreased visual acuity of 0.16 . We performed a capsulotomy using a neodymium-YAG laser with a total energy of $56 \mathrm{~mJ}$ (Fig. 1). Five days later the patient noticed visual field defects in his left eye, which had a visual acuity of 0.8 and an intraocular pressure of $15 \mathrm{mmHg}$. No intraocular inflammation was seen immediately or 5 days after laser surgery; the lens was located intracapsularly without any distortion. We found choroidal effusion of the nasal and temporal fundus, adjacent to the posterior pole (Fig. 2). Fortunately 2 weeks later the choroidal effusion had resolved spontaneously.

\section{Comment}

Spontaneous choroidal effusion without preceding ocular surgery can occur in nanophthalmos, perhaps due

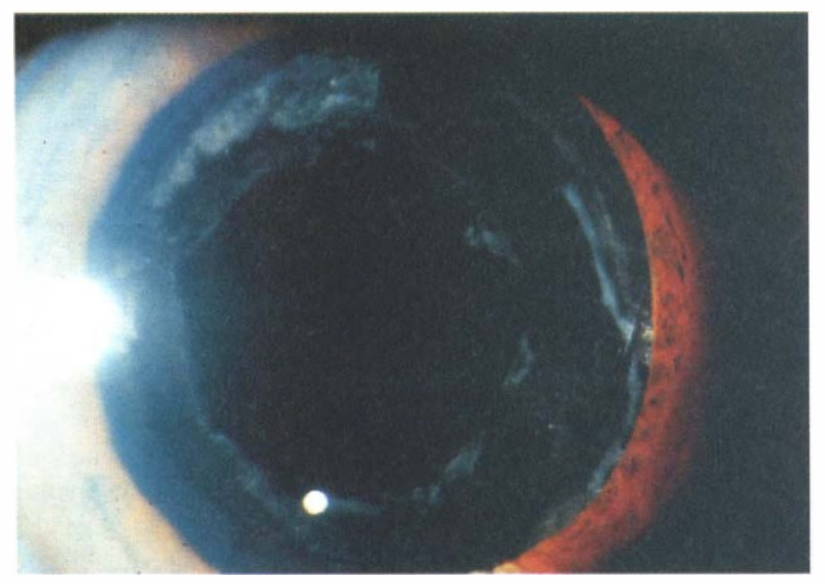

Fig. 1. Left eye: status after capsulotomy using a neodymium-YAG laser, 5 days after treatment.

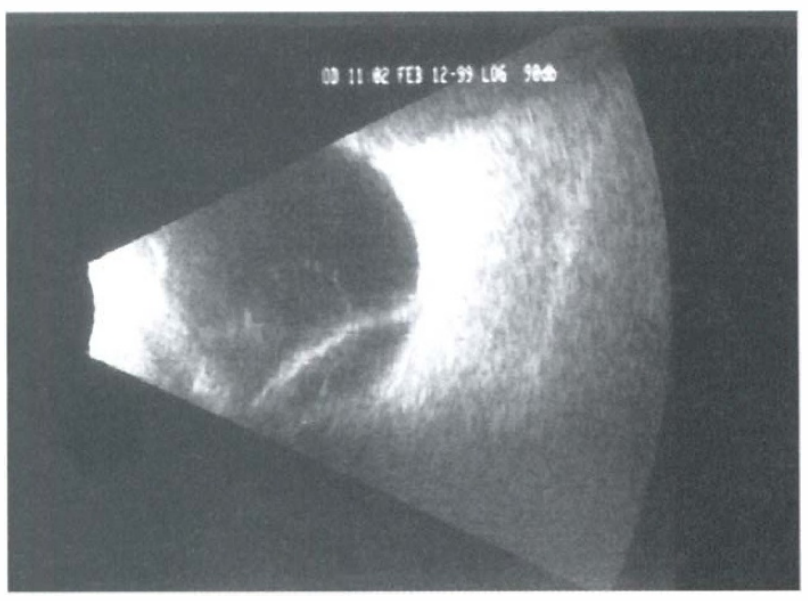

Fig. 2. Left eye: B-scan ultrasonography of the left eye. A peripheral choroidal detachment is seen in the nasal lower meridian.

to a relative obstruction to venous outflow via the vortex veins. ${ }^{3}$ The eye length of our patient was $25.33 \mathrm{~mm}$, so nanophthalmos could be ruled out. The only predisposition for our patient is his age, since the vascular system of older individuals is more likely to be incompetent. ${ }^{1}$ The intraocular lens (IOL) in our patient was located within the capsular bag without any distortion or capsule contraction. Lanzl and Kopp ${ }^{4}$ reported a case with ciliary body detachment caused by capsule contraction following phacoemulsification with IOL implantation.

We assume a causal relation between the two events since the choroidal effusion occurred 5 days after YAG laser capsulotomy. The question arises how YAG laser capsulotomy could have led to a choroidal effusion. We had no evidence of intraocular inflammation 1 or 5 days after the laser surgery was performed. It is hard to assume that only minor intraocular inflammation could have induced vascular leakage leading to choroidal effusion of such a considerable extent. The laser energy used was within the normal range, and thus we do not assume a correlation between the choroidal effusion and the amount of energy being used. Treatment is not absolutely necessary, as was demonstrated in our patient, in whom the choroidal effusion disappeared spontaneously within 2 weeks.

To the best of our knowledge no case of choroidal effusion after capsulotomy with a YAG laser has been described before. The pathogenesis remains unclear.

\section{References}

1. Albert DM, Jakobiec FA, editors. Principles and practice of ophthalmology: clinical practice, vol 3. Philadelphia: WB Saunders, 1994:549-59.

2. Huamonte FU, Peyman GA, Goldberg MF, et al. Immediate fundus complications after retinal scatter photocoagulation. I. Clinical picture and pathogenesis. Ophthalmic Surg 1976;7:88.

3. Weiter JJ, Brockhurst RJ, Tolentino FI. Uveal effusion following pan-retinal photocoagulation. Ann Ophthalmol 1979;11:1723.

4. Lanzl IM, Kopp C. Ciliary body detachment caused by capsule contraction. J Cataract Refract Surg 1999;25:1412-4. 


\section{Sir,}

\section{Congenital absence of the inferior rectus muscle in a patient with neurofibromatosis}

Congenital absence of extraocular muscle is a rare cause of strabismus. Involvement of the superior oblique tendon is the most frequently reported, ${ }^{1,2}$ although rectus muscle involvement also occurs. ${ }^{3-6}$ Recently, a case of congenital absence of the superior oblique tendon was reported in a patient with neurofibromatosis type 1 (NF $1){ }^{7}$ We report a case of congenital absence of the inferior rectus muscle, also in a patient with NF 1 . Congenital absence of extraocular muscle(s) has not been a previously recognised association of this condition.

\section{Case report}

A 12-year-old white female with a history of NF 1 was referred to our unit with a complaint of being teased at school due to a cosmetically poor vertical squint. Her diagnosis of neurofibromatosis was based on a family history of the condition, along with multiple café-au-lait spots, axillary freckles, a cutaneous neurofibroma and learning difficulties. She had had a left hypertropia since birth and had been treated for amblyopia with occlusion therapy, without spectacle correction and without success. There was no history of previous eye surgery and she was otherwise well and receiving no medications.

On examination, her unaided visual acuity was $6 / 9$ N5 in the right eye and 6/60 N16 in the left eye with no pinhole improvement. She had no evidence of binocular vision and demonstrated manifest latent horizontal nystagmus in her left eye. The left hypertropia measured 20 prism dioptres in the primary position (Fig. 1), decreasing in right gaze and increasing to 35 prism dioptres in left gaze. She also had left lower lid retraction. Versions demonstrated marked underaction of the left inferior rectus (Fig. 2) and surgery was planned to resect the left inferior rectus without dissection of the lower lid retractors.

At the time of surgery, forced duction testing revealed no restrictions and there was no evidence of previous surgery. An inferior conjunctival incision was made, but the inferior rectus muscle was not identified. The conjunctival incision was extended to expose the inferior half of the globe. The inferior oblique muscle was identified and followed but still the inferior rectus muscle could not be found. It was therefore concluded to be absent. Normal superior, medial and lateral rectus

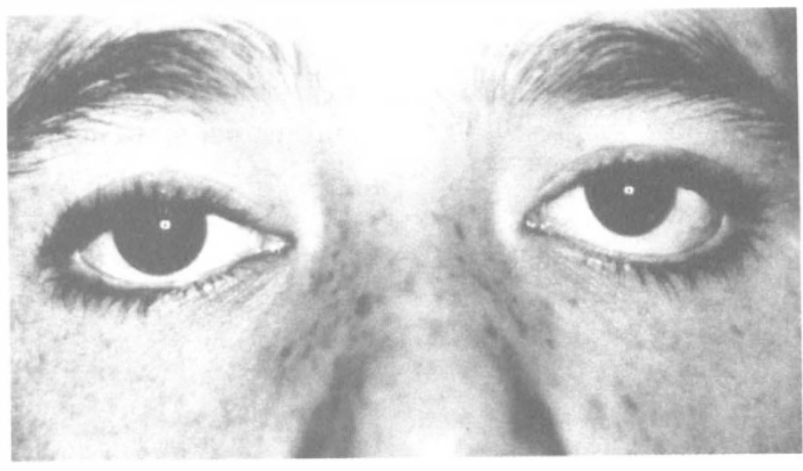

Fig. 1. Photograph with gaze in the primary position showing a left hypertropia and lower lid retraction.

muscles were identified, including normal ciliary vessels arising from the muscles. The superior oblique muscle was also normal. After due consideration of the risk of anterior segment ischaemia, a $7 \mathrm{~mm}$ recession of the left superior rectus muscle was performed.

Post-operatively, there was increased depression of the left eye with no deviation in the primary position and a good cosmetic result. A computed tomography scan of the orbits revealed no bony defects.

\section{Comment}

Congenital absence of one or more extraocular muscles is a rare entity. There is a known association with the syndromes of premature cranial bone stenosis (e.g. craniofacial dysostosis), ${ }^{8}$ thus linking cranial bone abnormalities with absent extraocular muscle. Abnormality of the orbital bones is also recognised in NF $1,{ }^{9}$ and most frequently consists of hypoplasia of the greater and lesser wings of the sphenoid bone, resulting in pulsating exophthalmos due to encephalocele.

The finding of congenital absence of the inferior rectus muscle and NF 1 in our patient may be a coincidence. However, this is the second report of absent extraocular muscle in a patient with NF 1. Although the mechanism is unclear and may differ between conditions, a link between cranial bone abnormalities and abnormal extraocular muscle exists and may account for the findings in our patient.

In 1971, Cooper and Greenspan ${ }^{3}$ reviewed all 7 previously reported cases and presented a further case of congenitally absent inferior rectus muscle. No cases had

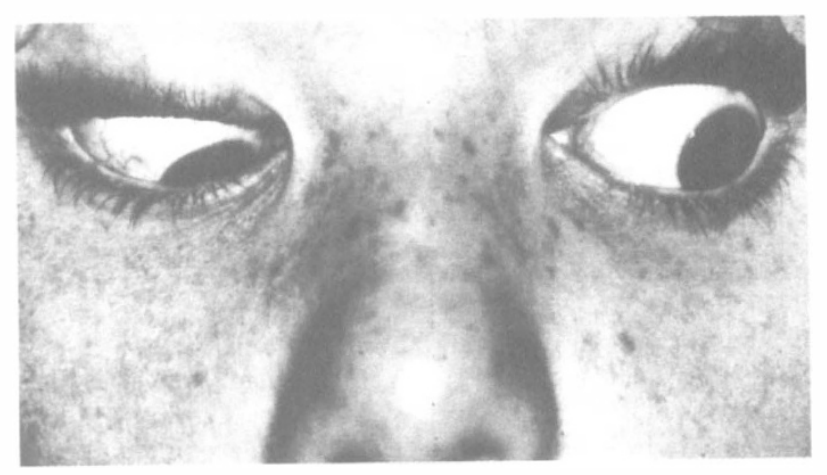

Fig. 2. Photograph with gaze in laevodepression showing marked underaction of the left inferior rectus. 